\title{
Assessment of Clinical Outcome Following Therapeutic Penetrating Keratoplasty in Non-Healing Infective and Perforated Corneal Ulcer
}

\author{
C. Suria Rashmi ${ }^{1}$, Gajaraj Tulsidas Naik², K. Satish ${ }^{3}$, Meghana Neeralgi ${ }^{4}$, Mohamed Abdul Kayoom5, \\ Sheetal Vaijanath Zille ${ }^{6}$, C. N. Madhusudhana ${ }^{7}$, Amulya Padmini H.M. ${ }^{8}$
}

1, 2, 3, 4, 5, 6, 7, 8 Department of Ophthalmology, Mysore Medical College and Research Institute, Mysore, Karnataka, India.

\section{ABSTRACT}

\section{BACKGROUND}

Corneal blindness has been a significant visual disability in developing countries like India. Corneal ulcer contributes mainly as the leading cause for monocular blindness. Considering the common condition, the studies and literature have been surprisingly less when it comes to corneal ulcer and their management. But with the advent of therapeutic penetrating keratoplasty (TPK), the scenario and prevalence of corneal blindness can be reduced. Hence this study was done to know the importance of therapeutic penetrating keratoplasty in case of non-healing infective and perforated corneal ulcers. The objectives were to assess the clinical outcome in a non-healing and perforated corneal ulcer, reduction in symptoms and signs of infection, anatomical or structural integrity of the globe and also evaluate the visual outcome following therapeutic penetrating keratoplasty.

\section{METHODS}

Data were obtained from 30 patients operated at our institute for therapeutic penetrating keratoplasty. Patients with less than one year of follow up, paediatric cases, PL negative cases were excluded. The outcome was assessed based on maintenance of structural integrity, reduction in infective load, improvement in visual acuity and graft survival and its correlation with corneal vascularisation, previously failed grafts, donor tissue quality, graft size and type of surgery.

\section{RESULTS}

Structural integrity was maintained in $93.3 \%$ of the cases, reduction of infective load in $90 \%$ of cases and optically clear grafts in $46.7 \%$ of cases and vision was better than $6 / 60$ in $30 \%$ of cases.

\section{CONCLUSIONS}

The study proved that in the case of non-healing and perforated corneal ulcers, therapeutic penetrating keratoplasty had a good prognosis in reducing infective load, maintaining structural integrity without which eye could have been lost.

\section{KEYWORDS}

Therapeutic Penetrating keratoplasty, Perforated corneal ulcer, non-healing corneal ulcer, corneal blindness, TPK
Corresponding Author:

Dr. C. Suria Rashmi,

Department of Ophthalmology,

Mysore Medical College and Research

Institute, Mysore, Karnataka, India.

E-mail: suria.chummi@gmail.com

DOI: $10.14260 / j e m d s / 2021 / 744$

How to Cite This Article:

Rashmi CS, Naik GT, Satish $K$, et al. Assessment of clinical outcome following therapeutic penetrating keratoplasty in non-healing infective and perforated corneal ulcer. J Evolution Med Dent Sci 2021;10(43):3670-3675, 10.14260/jemds/2021/744

Submission 29-07-2021,

Peer Review 10-10-2021,

Acceptance 16-10-2021,

Published 25-10-2021.

Copyright (C) 2021 C. Suria Rashmi et al. This is an open access article distributed under Creative Commons Attribution License [Attribution 4.0 International (CC BY 4.0)] 


\section{BACKGROUND}

Blindness has been a major health issue in India since its independence. To tackle it, the government introduced National Programme for Control of Blindness in 1976 which was a $100 \%$ central scheme to reduce blindness to $0.3 \%$.

The main causes of blindness are cataract, refractive error, glaucoma, posterior segment pathologies etc. These compromise about $99 \%$ of blindness and $0.99 \%$ was due to corneal blindness.

Apart from cataract and refractive errors the next major cause of blindness in developing countries is corneal blindness, but depends on geographic area, health services and other factors to make it more prevalent. ${ }^{1}$

Corneal ulceration in developing countries has a great impact on morbidity and has been called a silent epidemic. ${ }^{2} \mathrm{~A}$ study done by Gonzales in the Madurai district of India showed 10-11 times more incidence of corneal ulcer when compared to Olmsted County in the US. ${ }^{3}$ The total occurrence of corneal ulcers in the same district was 850000 per year which was thirty times that of US. ${ }^{3}$ Linking the prevalence of corneal ulcers in India and other Asian and African countries the total count goes over 2 million. In the end, majority of cases result in corneal blindness or even worse like endophthalmitis and phthisical eye.

Bacterial keratitis is the most common cause of infective keratitis and a common cause of perforation. If the perforations are very large that can't be plugged by tissue adhesives or glues they need keratoplasty. ${ }^{4}$ Corneal transplantation, where a diseased corneal tissue is replaced by donor corneal tissue in its entirety (penetrating) or in part (lamellar) is called keratoplasty. The various types of transplants are therapeutic, tectonic or reconstructive and optical.

We wanted to study the various aspects of therapeutic penetrating keratoplasty, including indications, pre-operative assessment, pre-operative infective load, surgical techniques, post-operative management, complications, post-operative reduction of infective load and post-operative structural and visual outcome. This procedure is done to eliminate the infection and re-establish the integrity of the globe with the best possible visual restoration. The study was done on patients, with non-healing and perforated corneal ulcers, attending OPD at KR Hospital, Mysore.

\section{Review of Literature}

Since the era of corneal transplantation, therapeutic keratoplasty has made wonders in the management of progressive corneal ulcers giving a $100 \%$ cure rate in the bacterial cause. But visual prognosis depends on a lot of factors such as the causative agent, inflammation, graft size, donor material quality. The main purpose of the surgery was to re-establish the globe integrity and eradicate the infectious disease process. The secondary outcome being visual prognosis. $^{5}$

The following are the results of therapeutic keratoplasty done at various institutes.

A 2-year retrospective study was done in an eye hospital. Pleven showed anatomy integrity was maintained in all the cases included in the study. The results say that TPK is best for perforated corneal ulcers. ${ }^{6}$

One more study done at Ankara Training and Research Hospital, Turkey showed a good prognosis after TPK in both the infectious and non-infectious keratitis groups which was conducted on 24 eyes for 2 years. 23 patients had Snellens more than $6 / 12$ after TPK. ${ }^{7}$

In a study done by $\mathrm{J} \mathrm{C}$ Hill, both perforated bacterial corneal ulcers and controls were included. The result being about half of the subjects had visual acuity of more than $6 / 12$ after TPK. ${ }^{8}$

In a study performed at Singapore National Eye Centre, more than $70 \%$ of the patients had a therapeutic survival rate after TPK for one year. ${ }^{9}$

\section{Objectives}

- To assess the reduction in signs and symptoms of infection following therapeutic penetrating keratoplasty.

- To assess the anatomical or structural integrity of the globe following therapeutic penetrating keratoplasty.

- To assess the visual outcome following therapeutic penetrating keratoplasty.

\section{METHODS}

The ethical and research committee of our hospital approved this study. Informed written consent was obtained from each participant. This was an interventional study conducted between January 2016 and June 2017.

\section{Sampling Method}

Purposive sampling method.

\section{Inclusion Criteria}

All patients undergoing therapeutic penetrating keratoplasty for non-healing ulcer, impending perforation and perforated corneal ulcer.

\section{Exclusion Criteria}

Non-healing corneal ulcer with endophthalmitis and PL negative individuals.

\section{Sample Size}

Estimated to be 30 subjects with formula.

$S=\frac{Z^{2} p q}{d^{2}}$

Where $\mathrm{P}$ (prevalence) $=0.88$, level of significance $=5 \%$, absolute allowable error $=12 \%$, using estimator set up technique for proportion, the inflated sample size was 30 . 


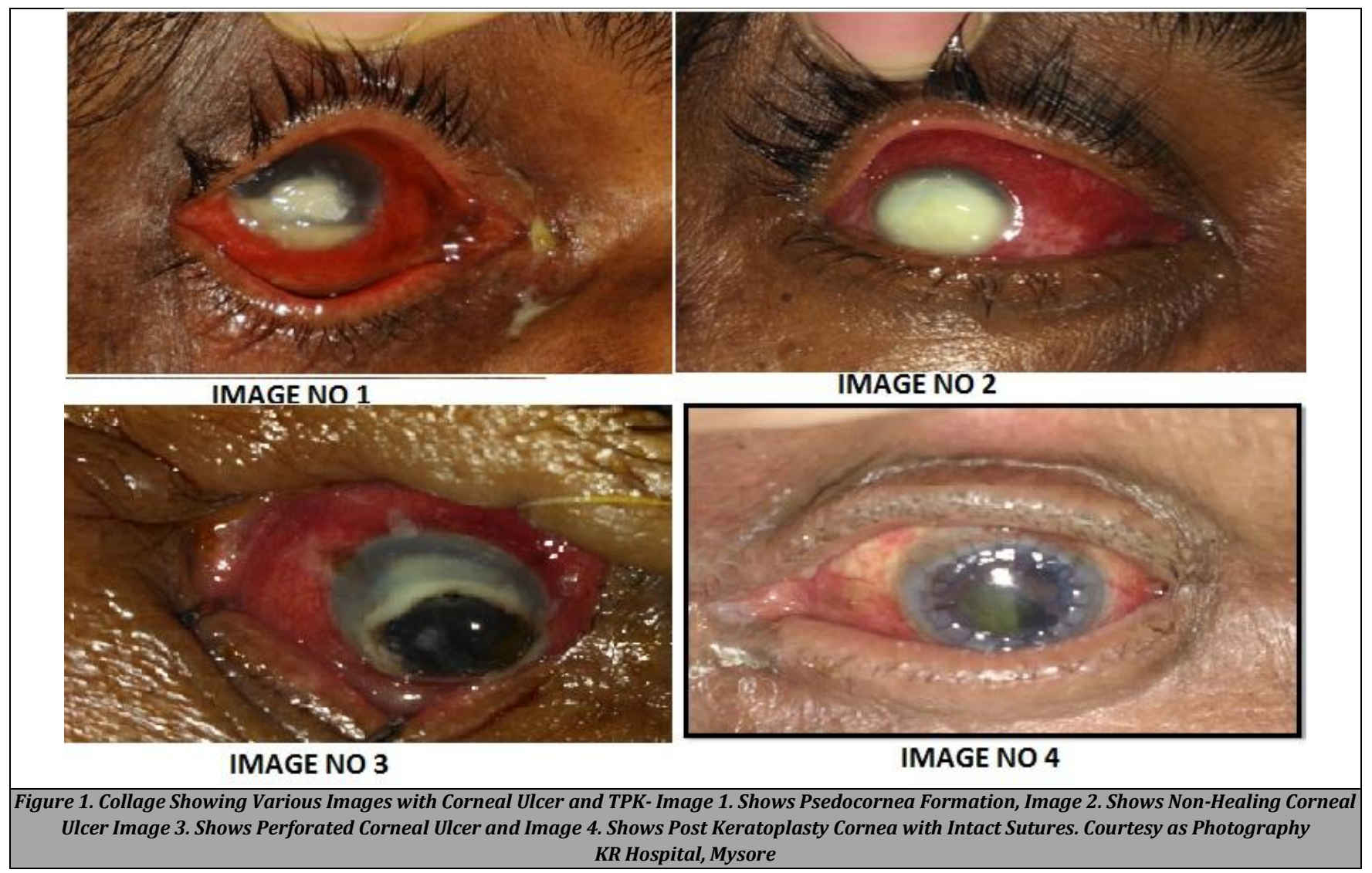

- Patients fulfilling the inclusion and exclusion criteria were explained about the condition and procedure and consent taken. Detailed examination including ocular and systemic was done in special proforma which was transferred to the master sheet. The data was processed for statistical analysis.

- Patient details such as name, age, sex, socio-economic status, occupation, chief complaints, history of presenting illness, the time duration between onset and presenting to hospital, prior antibiotic treatment were collected.

- Ophthalmic examination was done to assess the state of the diseased eye - site, size and depth of the ulcer, size and site of perforation or impending perforation, vascularisation of the cornea, scleral involvement, the inflammatory status of the eye and hypopyon.

- B scan of the affected eye was done to assess any posterior segment pathology. Detailed examination was done to assess the causative agent (bacteria, fungi, viral, acanthamoeba) by corneal scrapings and microbiological examination by the microbiologist at KR Hospital.

- General examination to assess the diabetic and hypertensive status of the patient was done.

- Patients were started on systemic broad-spectrum antibiotics and topical antibiotics and antifungal and cycloplegics.

- Examination of donor tissue for size, quality of the graft and grading of donor cornea was done.

- Therapeutic penetrating keratoplasty was performed under peribulbar anaesthesia or general anaesthesia by the ophthalmic surgeon.
- Postoperative medications were continued and follow up examination on post-op day 1 , day 7 , day 15 , day 30 , thereafter monthly up to 1 year was done.

- Post-op slit-lamp examination to assess the reduction in signs and symptoms, reduction in infective load, graft uptake, wound leak, graft transparency, visual acuity was done.

\section{Statistical Methods Applied}

Statistical analysis included descriptive statistics, inferential statistics, chi-square test and Cramer's V test. SPSS software version 16.0 was used.

\section{RESULTS}

\begin{tabular}{|cccc|}
\hline Age Distribution in Years & Number of Patients & Male Sex & Female Sex \\
$<40$ & 3 & 1 & 2 \\
$41-50$ & 4 & 2 & 2 \\
$51-60$ & 11 & 07 & 4 \\
$60+$ & 12 & 07 & 05 \\
Total & $\mathbf{3 0}$ & $\mathbf{1 7}$ & $\mathbf{1 3}$ \\
\hline \multicolumn{4}{c}{ Table 1. Age and Sex Distribution } \\
\hline
\end{tabular}

\begin{tabular}{|ccc|}
\hline $\begin{array}{c}\text { Structural Integrity } \\
\text { Maintained }\end{array}$ & Number of Patients & Percentage \\
No & 2 & 6.7 \\
Yes & 28 & 93.3 \\
Total & $\mathbf{3 0}$ & $\mathbf{1 0 0 . 0}$ \\
\hline Table 2. Post Op Maintenance of Structural Integrity \\
\hline
\end{tabular}

\begin{tabular}{|ccc|}
\hline Infective Load & No of Patients & Percentage \\
Reduced & 27 & 90.0 \\
Reinfection & 3 & 10.0 \\
Total & $\mathbf{3 0}$ & $\mathbf{1 0 0 . 0}$ \\
\hline Table 3. Post Op Reduction in Infective Load \\
\hline
\end{tabular}




\begin{tabular}{|ccc|}
\hline Graft Outcome & $\begin{array}{c}\text { Number of } \\
\text { Patients }\end{array}$ & Percentage \\
\hline Optically clear & 14 & 46.7 \\
Significant haze & 9 & 30.0 \\
Opaque grafts & 5 & 16.7 \\
Evisceration & 2 & 6.7 \\
Total & $\mathbf{3 0}$ & $\mathbf{1 0 0 . 0}$ \\
\hline Table 4. Outcome in Terms of Graft Clarity \\
\hline
\end{tabular}

\begin{tabular}{|cccc|}
\hline Graft Outcome & \multicolumn{3}{c|}{ Corneal Periphery } \\
Healthy & Involved & Total \\
Optically clear & 8 & 6 & 14 \\
Significant haze & 2 & 7 & 9 \\
Opaque grafts & 1 & 4 & 5 \\
evisceration & 0 & 2 & 2 \\
Total & $\mathbf{1 1}$ & $\mathbf{1 9}$ & $\mathbf{3 0}$ \\
\hline Table 5. Involvement of Corneal Periphery Versus Graft Outcome \\
\hline
\end{tabular}

\begin{tabular}{|ccccccc|}
\hline $\begin{array}{c}\text { Graft } \\
\text { Outcome }\end{array}$ & Nil & $\begin{array}{c}\mathbf{1} \\
\text { Quadrant }\end{array}$ & $\begin{array}{c}\mathbf{2} \\
\text { Quadrant Quadrant }\end{array}$ & $\mathbf{3}$ & $\mathbf{4}$ & Total \\
Optically clear & 6 & 4 & 4 & 0 & 0 & 14 \\
Significant haze & 1 & 2 & 3 & 3 & 0 & 9 \\
Opaque graft & 0 & 1 & 1 & 3 & 0 & 5 \\
Evisceration & 1 & 0 & 0 & 0 & 1 & 2 \\
Total & $\mathbf{8}$ & $\mathbf{7}$ & $\mathbf{8}$ & $\mathbf{6}$ & $\mathbf{1}$ & $\mathbf{3 0}$ \\
\hline \multicolumn{7}{|c|}{ Table 6. Vascularization of Host Versus Graft Outcome } \\
\hline
\end{tabular}

\begin{tabular}{|c|cccccc|}
\hline Graft Outcome & $\mathbf{7 . 5 0}$ & $\mathbf{8 . 0 0}$ & $\mathbf{8 . 5 0}$ & $\mathbf{9 . 0 0}$ & $\mathbf{9 . 5 0}$ & Total \\
Optically clear & 1 & 1 & 5 & 6 & 1 & 14 \\
Significant haze & 0 & 1 & 2 & 3 & 3 & 9 \\
Opaque graft & 0 & 0 & 0 & 4 & 1 & 5 \\
Evisceration & 0 & 0 & 0 & 0 & 2 & 2 \\
Total & $\mathbf{1}$ & $\mathbf{2}$ & $\mathbf{7}$ & $\mathbf{1 3}$ & $\mathbf{7}$ & $\mathbf{3 0}$ \\
\hline Table 7. Graft Size Versus Graft Outcome \\
\hline
\end{tabular}

\begin{tabular}{|c|c|c|c|}
\hline \multirow{2}{*}{ Graft Outcome } & \multicolumn{2}{|c|}{ Donor Grade } & \multirow{2}{*}{ Total } \\
\hline & A & B & \\
\hline Opaque graft & 8 & 6 & 14 \\
\hline Significant haze & 2 & 7 & 9 \\
\hline Opaque grafts & 2 & 3 & 5 \\
\hline Evisceration & 2 & 0 & 2 \\
\hline Total & 14 & 16 & 30 \\
\hline \multicolumn{4}{|c|}{ Table 8. Graft Grading Versus Graft Outcome } \\
\hline
\end{tabular}

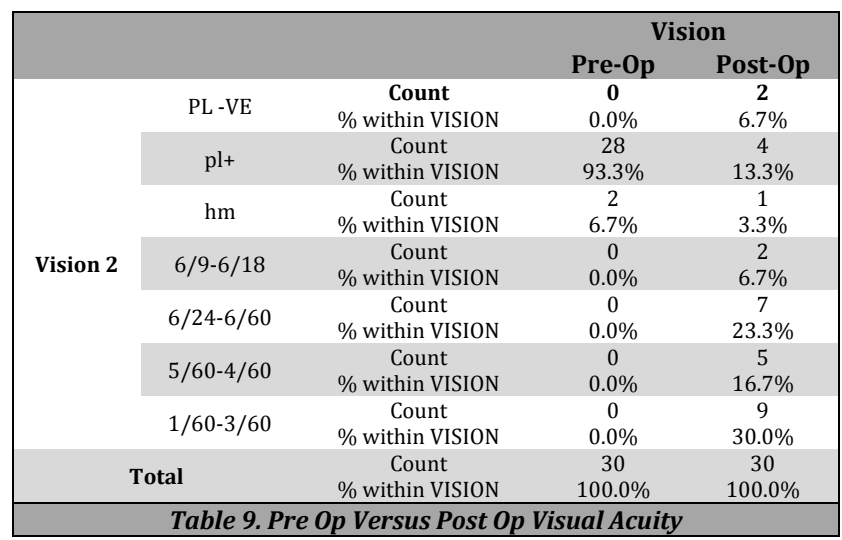

Of the 30 patients, 17 (56.7\%) were males and 13 (43.3 $\%)$ were females. 3 patients $(10 \%)$ were $<40$ years of age, 4 patients (13\%) were in $41-50$ years of age and 11 patients (36.7\%) in 51 - 60 years of age and 12 patients (40\%) were more than 60 years of age.

10 patients (33.3\%) were diagnosed with a chronic nonhealing ulcer, 9 patients (30\%) were diagnosed with a corneal ulcer with impending perforation, 6 patients $(20 \%)$ with perforated corneal ulcer and 5 patients $(16.7 \%)$ with pseudocornea.

Corneal scrapings showed staphylococci in 9 patients, streptococci, E. coli and acinetobacter in 2 patients each, fusarium in 3 patients, pseudomonas, aspergillus and candid in 1 patient each and 9 patients showed no growth.

Among the patients included in the study, 21 cases (70 \%) presented with a corneal ulcer with hypopyon.

11 patients had the clear lens, 13 patients had a cataract lens and 6 patients had an intraocular lens. Those patients with cataract underwent a triple procedure, which is lens extraction anterior vitrectomy if required, PCIOL implantation in the bag or sulcus followed by therapeutic penetrating keratoplasty.

Other factors such as involvement of corneal periphery, presence or absence of vascularisation and the number of quadrants involved and the presence or absence of peripheral anterior synechiae were all noted and compared with the post-op outcome.

Pre-op vision in all the patients ranged from hand movement to PL positive

Following penetrating keratoplasty, structural integrity was maintained in 28 eyes (93.3\%) and 2 patients had to undergo evisceration due to re-infection, hence in $6.7 \%$ of patients maintenance of anatomical structural integrity was not possible.

Following penetrating keratoplasty, reduction in infective load was achieved in 27 eyes $(90 \%)$ and 3 patients (10\%) had re-infection. Of the 3 patients who had re-infection, one patient underwent repeat keratoplasty and the structural integrity was maintained, whereas 2 patients underwent evisceration.

At the end of 1 year of follow up, 14 eyes (46.7\%) had optically clear grafts, 9 eyes (30\%) had significantly hazy grafts, 5 eyes (16.7\%) had opaque grafts and evisceration was done in 2 eyes (6.7\%). P-value was 0.013 , which was statistically significant, hence following penetrating keratoplasty, post-op graft outcome was favourable.

The extent of corneal periphery involvement and scleral involvement correlates strongly with the graft outcome. Corneal periphery was involved in 19 out of 30 cases and of which the results were as follows. 6 eyes had clear grafts, 7 patients developed significant haze, 4 patients had opaque grafts and 2 eyes that were eviscerated due to re-infection also had corneal peripheral involvement. P-value was 0.165 ; although not statistically significant, a trend could be observed in the results wherein of 11 patients without corneal peripheral involvement, 8 patients had optically clear grafts.

Of 8 cases with no vascularization, 6 cases had optically clear grafts, of 7 cases with 1 quadrant vascularization, 4 cases had clear grafts, 2 cases with significant haze and 1 case with opaque graft, of 8 cases with 2 quadrant vascularization, 4 cases had optically clear grafts and 3 cases had significant haze, of 6 cases with 3 quadrant vascularisation, 3 cases had a significant haze and 3 cases had optically opaque grafts, 1 case that had all 4 quadrants vascularized underwent reinfection and had to be eviscerated.

A P-value of 0.007 was statistically significant.

The above figure shows that the presence of PAS causes poorer outcomes due to secondary glaucoma leading to graft failure and hence a majority of cases with PAS had developed significant haze or opaque grafts.

Although P-value being 0.210 is not statistically significant, eyes with PAS have had a poor outcome. 
The figures in the table clearly show that optically clear grafts were obtained with smaller graft sizes $(7.50 \mathrm{~mm}$ to $8.50 \mathrm{~mm})$. Larger grafts were taken in about 20 cases $(9.0$ $\mathrm{mm}$ to $9.50 \mathrm{~mm}$ ) of which 13 eyes had developed significant haze to opaque grafts.

$\mathrm{P}$-value was statistically insignificant $(\mathrm{P}=0.295)$

Donor graft was graded based on slit-lamp examination. In our study, optical clear grafts were seen in 8 eyes that used A grade grafts, as compared with B grade cornea where 7 cases had developed significant haze, but this difference was not statistically significant $(P=0.161)$

At the time of diagnosis, $93.3 \%$ (28 patients) had a visual acuity of PL positive and $6.7 \%$ ( 2 cases) had visual acuity as hand movements.

\section{DISCUSSION}

Corneal transplant surgery is the most commonly performed allograft worldwide and also most successful with the high survival rate of graft. However, long term success rate decreases according to Australian corneal graft registry (ACGR). The survival rates of grafts are $79.6 \%, 68.7 \%$, and $46.5 \%$ after 1,2 , and 5 years respectively according to studies done in India. This in comparison to western studies is quite different with regard to so many factors like patient presentation, storage of graft tissue, socioeconomic status etc.

In our study, we have considered only therapeutic penetrating keratoplasty for 30 patients whose outcome has been studied at the end of 1 year of follow-up. The study sample had patients with age groups ranging from 30 years to 70 years, but $76 \%$ of patients ranged between the age group of 50 and 70 years. Males constituted $56.7 \%$ and females $43.3 \%$. Hence males over the age of 50 were at a higher risk of developing a corneal ulcer. A study done by Keshav BR et al. had similar results. ${ }^{10}$ As per Jones guidelines, a slit lamp examination of the anterior segment was performed in all patients. The findings noted were size, shape and depth of ulcer extent of infiltrates, hypopyon, scleral involvement and corneal complications. In the end, grading was done. Patients included in the study group either had a chronic non-healing corneal ulcer or impending perforation or pseudocornea or a perforated corneal ulcer.

Corneal scrapings were done and sent for culture sensitivity. In our study, the most common bacteria isolated were staphylococci and the most common fungi were fusarium. About 10 percent of the cases showed no growth the reason being improper scraping or contamination.

$43.3 \%$ of the study population had a co-existing cataract at the time of presentation and hence underwent triple procedure wherein lens extraction with open sky vitrectomy with PCIOL implantation in the sulcus followed by penetrating keratoplasty was done.

At the end of 1 year of follow up, structural integrity was maintained in $93.3 \%$ of the patients and $6.7 \%$ of the patients had to undergo evisceration due to re-infection. Reduction of the infective load was achieved in $90 \%$ of the cases and reinfection in $10 \%$ of the cases.

TPK has success in maintaining structural integrity and stabilization of the eye according to many studies. ${ }^{11}$

Optically clear grafts were obtained in $46.7 \%$ of the cases, significant haze in $30 \%$ of cases, opaque grafts in 16.7 $\%$ of cases and evisceration in $6.7 \%$ cases. These results are consistent with the literature. In one study by Nurozler, the final VA was 0.2 or better in $60 \%$ of perforated corneal diseases after surgery with clear grafts. ${ }^{12}$ One more study by Gong, showed that the final VA was 0.005 or better in more than 15 cases in case of suppurative corneal ulcer. ${ }^{13}$

Pre-op vision in all the cases ranged from PL positive to hand movements and the post-op vision at the end of 1 year was as follows. $6.7 \%$ had a BCVA of $6 / 9$ to $6 / 18,23.3 \%$ had a BCVA of $6 / 24$ to $6 / 60,16.7 \%$ had a BCVA of $5 / 60$ to $4 / 60$, $30.0 \%$ had a BCVA of $1 / 60$ to $3 / 60,3.3 \%$ had BCVA of hand movements, $13.3 \%$ had BCVA of PL positive, $6.7 \%$ who underwent evisceration had PL negative.

In our analysis, we found that of 8 cases with no vascularization 6 cases had optically clear grafts, of 7 cases with 1 quadrant vascularization 4 cases had clear grafts, 2 cases with significant haze and 1 case with opaque graft, of 8 cases with 2 quadrant vascularization 4 cases had optically clear grafts and 3 cases had significant haze, of 6 cases with 3 quadrant vascularization 3 cases had a significant haze and 3 cases had optically opaque grafts, 1 case that had all 4 quadrants vascularized underwent re-infection and had to be eviscerated. Many studies have shown that the graft survival was less in the case of vascularized. ${ }^{14,15}$

Other factors such as corneal periphery involvement, presence or absence of PAS, graft size and graft grading were also compared with the outcome. Although none of these had a statistically significant p-value, our study shows that the involvement of corneal periphery and presence of PAS had more number of opaque grafts. Since only A and B grade corneas were used in the procedure, significant differences were not seen in comparison with the outcome. Smaller grafts showed better outcomes than larger grafts.

\section{CONCLUSIONS}

Our results confirm that to restore the anatomic integrity of the eye and visual rehabilitation in case of perforated corneal ulcer, therapeutic keratoplasty has a major role. So TPK has been a necessary surgery in preventing corneal blindness worldwide.

Data sharing statement provided by the authors is available with the full text of this article at jemds.com.

Financial or other competing interests: None.

Disclosure forms provided by the authors are available with the full text of this article at jemds.com.

\section{REFERENCES}

[1] Smith GT, Taylor HR. Epidemiology of corneal blindness in developing countries. Refract Corneal Surg 1991;7(6):436-9.

[2] Whitcher JP, Srinivasan M. Corneal ulceration in the developing world--a silent epidemic. Br J Ophthalmol 1997;81(8):622-3.

[3] Gonzales CA, Srinivasan M, Whitcher JP, et al. Incidence of corneal ulceration in Madurai District, South India. Ophthal Epidemiol 1996;3(3):159-66. 
[4] Murillo-Lopez F. Keratitis, Bacterial. Emedicine April 18, 2006.

[5] Sony P, Sharma N, Vajpayee RB, et al. Therapeutic keratoplasty for infectious keratitis: a review of the literature. CLAO J 2002;28(3):111-8.

[6] Balabanov C, Murgova S, Parashkevova B, et al. Treatment of perforated infectious corneal ulcers with penetrating keratoplasty. Journal of IMAB 2007;13(1):35-9.

[7] Yalniz-Akkaya Z, Burcu A, Dogan E, et al. Therapeutic penetrating keratoplastyfor infections and noninfectious corneal ulcers. Int Ophthalmol 2015;35(2)193-200.

[8] McLeod SD, Kolahdouz-Isfahani A, Rostamian K, et al. The role of smears, cultures and antibiotic sensitivity testing in the management of suspected infectious keratitis. Ophthalmology 1996;103(1):23-8.

[9] Ti SE, Scott AJ, Janardhan P, et al. Therapeutic keratoplasty for advanced suppurative Keratitis. Am J Ophthalmol 2007;143(5):755-62.
[10] Keshav BR, Zacheria G, Ideculla T, et al. Epidemiological characteristics of corneal ulcers in south Sharqiya region, Oman Med J 2008;23(1):334-9

[11] Raj A, Gupta N, Dhasmana R, et al. Indications and visual outcome of penetrating keratoplasty in Tertiary Eye Care Institute in Uttarakhand. J Clin Diagn Res 2016;10(6):NC01-4.

[12] Nurozler AB, Salvarli S, Budak K, et al. Results of therapeutic penetrating keratoplasty. Jpn J Ophthalmol 2004;48(4):368-71.

[13] Gong XM, Chen JQ, Feng CM. Penetrating keratoplasty in the treatment of corneal perforation. Zhonghua Yan Ke Za Zhi 1994;30(1):8-10.

[14] Tan DT, Janardhanan P, Zhou H, et al. Penetrating keratoplasty in asian eyes: the Singapore corneal transplant study. Ophthalmology 2008;115(6):975-82 e1.

[15] Price MO, Thompson RW, Price FW. Risk factors for various causes of failure in initial corneal grafts. Arch Ophthalmol 2003;121(8):1087-92. 